\title{
Zigzag Zinc Blende ZnS Nanowires: Large Scale Synthesis and Their Structure Evolution Induced by Electron Irradiation
}

\author{
Daesoo $\mathrm{Kim}^{\S}$, Paresh Shimpi ${ }^{\S}$, and Pu-Xian Gao $(\bowtie)$ \\ Department of Chemical, Materials and Biomolecular Engineering \& Institute of Material Science, University of Connecticut, Storrs, \\ CT 06269-3136, USA
}

Received: 20 September 2009 / Revised: 23 October 2009 / Accepted: 23 October 2009

(C) Tsinghua University Press and Springer-Verlag 2009. This article is published with open access at Springerlink.com

\begin{abstract}
Large scale zigzag zinc blende single crystal ZnS nanowires have been successfully synthesized during a vapor phase growth process together with a small yield of straight wurtzite single crystal ZnS nanowires. AuPd alloy nanoparticles were utilized to catalyze a vapor-solid-solid growth process of both types of ZnS nanowires, instead of the more common vapor-liquid-solid growth process. Surprisingly, the vapor-phase grown zigzag zinc blende ZnS nanowires are metastable under high-energy electron irradiation in a transmission electron microscope, with straight wurtzite nanowires being much more stable. Upon exposure to electron irradiation, a wurtzite $\mathrm{ZnO}$ nanoparticle layer formed on the zigzag zinc blende $\mathrm{ZnS}$ nanowire surface with concomitant displacement damage. Both electron inelastic scattering and surface oxidation as a result of electron-beam heating occur during this structure evolution process. When prolonged higher-voltage electron irradiation was applied, local zinc blende ZnS nanowire bodies evolved into ZnS-ZnO nanocables, and dispersed ZnS$\mathrm{ZnO}$ nanoparticle networks. Random AuPd nanoparticles were observed distributed on zigzag ZnS nanowire surfaces, which might be responsible for a catalytic oxidation effect and speed up the surface oxidation-induced structure evolution.
\end{abstract}

\section{KEYWORDS}

ZnS nanowire, polymorph, vapor-solid-solid growth, transmission electron microscopy, electron irradiation, structure evolution

\section{Introduction}

The discovery of carbon nanotubes [1] has stimulated extensive research effort to search for and understand, both physically and chemically, a variety of one-dimensional functional nanostructures. Wide bandgap semiconductors like $\mathrm{ZnO}$ [2], GaN [3], and AlN [4], have been widely investigated as promising electronic and optoelectronic nanomaterials. In the case of $\mathrm{ZnS}$, a diverse array of growth morphologies such as nanowires [5-16], nanobelts [7, 12], nanocombs [9], and nanohelices [9] have

Address correspondence to puxian.gao@ims.uconn.edu

$\S$ These authors equally contributed to this work. 
been synthesized using vapor deposition processes. At ambient conditions, ZnS shows two structural polymorphs: wurtzite (hexagonal) and zinc blende (cubic). Wurtzite ZnS and zinc blende $\mathrm{ZnS}$ have band gaps of $3.91 \mathrm{eV}$ and $3.5 \mathrm{eV}$ [2] and are normally stable at low temperature and high temperature, respectively. The structure transformation has been suggested to occur above $1000{ }^{\circ} \mathrm{C}$ in the bulk form, and at $\sim 750{ }^{\circ} \mathrm{C}$ for single crystal ZnS nanobelts [17]. It has also been reported that $\mathrm{ZnS}$ nanoparticles with the zinc blende structure transformed into the cubic $\mathrm{NaCl}$ structure under high pressure, and that the transition was reversed when the pressure was released [18].

Although wurtzite-structured $\mathrm{ZnS}$ is believed to be a metastable phase at room temperature, most of the reported vapor phase deposited $\mathrm{ZnS}$ nanowires and nanobelts have been wurtzite structures $[7,12$, 17], with very few reports of predominantly zinc blende-structured ZnS nanowires [19] or nanobelts.
It has also been reported that vapor phase grown wurtzite-structured ZnS nanobelts are very robust under electron irradiation in a transmission electron microscope (TEM) [20] despite being the metastable phase at low temperature. In contrast, wurtzitestructured $\mathrm{ZnS}$ nanotubes grown using wet chemistry have been found to be extremely unstable under electron beam irradiation, which was attributed to the defective structures resulting from the lowtemperature aqueous processing [21].

In this study, we report the synthesis of large scale zigzag zinc blende single crystal $\mathrm{ZnS}$ nanowires using a AuPd catalyzed vapor-solid-solid growth process in the temperature range $\sim 700-900{ }^{\circ} \mathrm{C}$. Although they might have been expected to be stable, the zinc blende $\mathrm{ZnS}$ nanowires turned out to be ultrasensitive to electron irradiation exposure, which led to a structure evolution from $\mathrm{ZnS}$ nanowires to $\mathrm{ZnS}-\mathrm{ZnO}$ nanocables, and then to nanoparticle networks facilitated by a displacement damage effect
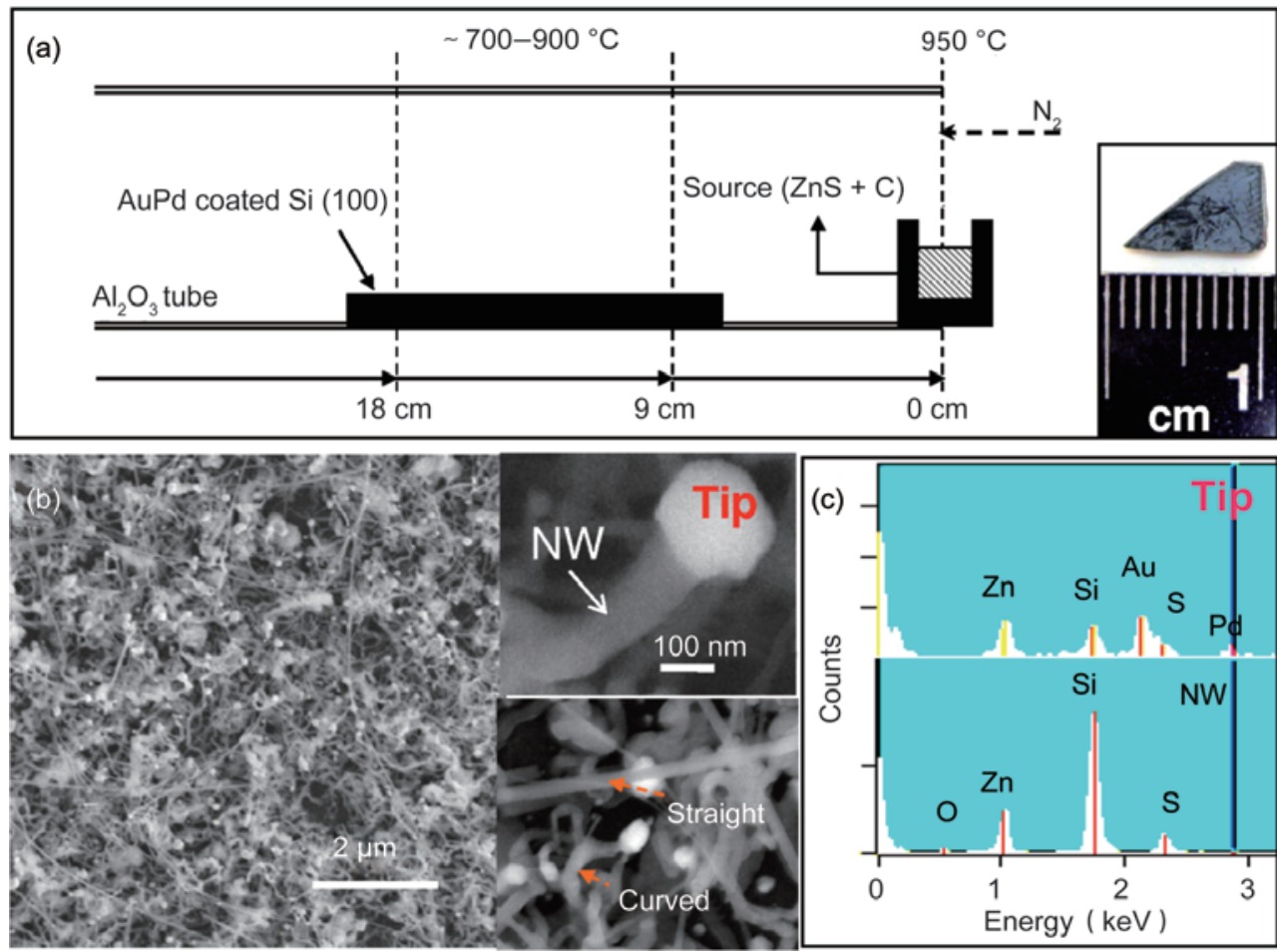

Figure 1 (a) A schematic diagram of substrate positions for ZnS nanowire deposition and an alumina boat loaded with source materials in the alumina tube. Right inset: a typical CCD image of a $1 \mathrm{~cm} \times 0.6 \mathrm{~cm} \mathrm{Si(100)}$ substrate deposited with ZnS nanowires. (b) A low-magnification SEM image showing ZnS nanowires (NW) grown on a AuPd-coated Si (100) substrate; insets: nanoparticle-tipped ZnS nanowire (top-right) and the observed straight and curved nanowires (bottom-right). (c) Typical EDX spectra showing the nanowire body containing Zn, S, with O and Si as impurities (bottom), and the tip containing Au and Pd (top) 
as well as an incomplete surface oxidation effect. Furthermore, AuPd nanoparticles have been found to be distributed on the $\mathrm{ZnS}$ nanowire surfaces, which could promote the surface oxidation process by increasing their surface oxygen adsorption ability under high vacuum.

\section{Experimental}

Commercial ZnS powder $(99.99 \%,<10 \mu \mathrm{m}$, Acros Organics) and graphite powder (Fisher Scientific) with a weight ratio of 2:1 were ground as the source material. The growth was conducted in a horizontal tube furnace attached to control systems for vacuum, carrier (reaction) gases and cooling. As shown in Fig. 1(a), the source loaded in an alumina boat was put in the tube furnace center and $\mathrm{Si}(100)$ substrates coated with $\sim 3-10 \mathrm{~nm}$ AuPd (Au:Pd = 40:60, weight percentage) films were put $\sim 9-18 \mathrm{~cm}$ downstream from the source boat. During the experiment, the furnace was pumped down to $\sim 5 \times 10^{-2}$ mbar, and was then heated to $\sim 950{ }^{\circ} \mathrm{C}$ with a ramping rate of $25^{\circ} \mathrm{C} / \mathrm{min}$ and held for $90 \mathrm{~min}$. The pressure was controlled at $\sim 100$ mbar during the heating process and $\mathrm{N}_{2}$ was used as carrier gas. During the cooling period, the carrier gas was stopped, the residual gas was purged and the chamber pressure was controlled at $\sim 5 \times 10^{-2}$ mbar. A JEOL JSM 6335F field emission scanning electron microscope (SEM), an FEI Tecnai 12 scanning transmission electron microscope (STEM), and a JEOL 2010 high-resolution TEM were used to characterize the morphology and structure of the nanowires. The composition was determined by energy dispersive X-ray (EDX) spectrometers attached to the SEM and TEM. An X-ray diffractometer (XRD, Bruker D5005) was used for determining the phase structures of nanowires grown on the substrates.

\section{Results and discussion}

Figure 1(a) schematically depicts the controlled deposition zones inside the tube furnace, with the source boat at the center of the alumina tube. The temperature of the deposition substrates $(9-18 \mathrm{~cm}$ from the boat) was controlled in the range $\sim 700$ $900{ }^{\circ} \mathrm{C}$ with the source boat temperature controlled at $\sim 950{ }^{\circ} \mathrm{C}$. The inset of Fig. 1(a) is a charge-coupled device (CCD) camera recorded image showing the surface of a $1 \mathrm{~cm} \times 0.6 \mathrm{~cm}$ substrate after deposition with uniformly grown $\mathrm{ZnS}$ nanowires. Figure 1(b) is a typical SEM image showing the morphology of ZnS nanowires formed in this temperature region. A large fraction of nanowires grown in this region (Fig. 1(b)) is randomly zigzag with diameters ranging from $\sim 50$ to $\sim 100 \mathrm{~nm}$ and lengths up to tens of micrometers. In addition, small amount of straight nanowires are also present, as indicated in the bottom-right inset in Fig. 1(b). Each curved nanowire has a nanoparticle tip on top. The top-right inset of Fig. 1(b) is a zoom-in SEM image showing the top portion of a single $\mathrm{ZnS}$ nanowire, which has a curved nanowire body and a nanoparticle tip. The EDX spectra collected from the tip and body part of this nanowire are displayed in Fig. 1(c). The presence of $\mathrm{Au}$ and $\mathrm{Pd}$ is clearly revealed in the tip spectrum (top), which confirms the catalytic function of the AuPd. From the bottom spectrum corresponding to the nanowire body, the atomic ratio of $\mathrm{Zn}$ and $\mathrm{S}$ is 50.4:49.6, closely matching the chemical stoichiometry of ZnS. It should also be noted that a small percentage of $\mathrm{O}$ is detected in the $\mathrm{ZnS}$ nanowires. The Si peaks in these spectra are due to the silicon wafer.

To determine the structure of the as-grown $\mathrm{ZnS}$ nanowires, X-ray diffraction and TEM imaging and diffraction analysis have been carried out. Figure 2(a) is a typical XRD spectrum scanned from the $\mathrm{ZnS}$ nanowires collected in the $\sim 700-900{ }^{\circ} \mathrm{C}$ zone. Wurtzite ZnS (JCPDS 36-1450, P63mc, $a=0.38209 \mathrm{~nm}$, $c=0.62573 \mathrm{~nm}$ ) can be identified, with other major peaks possibly resulting from overlapping wurtzite and zinc blende ZnS (JCPDS 5-566, F-43m $a=0.5406$ $\mathrm{nm})$ reflections $\left((002)_{\mathrm{w}} /(111)_{\mathrm{z}}\right.$ and $(110)_{\mathrm{w}} /(220)_{\mathrm{z}} \mathrm{w}$ : wurtzite, z: zinc blende), as indexed in the pattern. A thorough selected area electron diffraction (SAED) analysis associated with a number of SAED patterns collected from $\mathrm{ZnS}$ nanowires suggested that the zigzag $\mathrm{ZnS}$ nanowires are mainly zinc blende in structure, while the straight nanowires have the wurtzite structure. The TEM image in Fig. 2(b) reveals the coexistence of the curved $\mathrm{ZnS}$ nanowires and straight ZnS nanowires, both of which clearly have nanoparticle tips $\sim 80-100 \mathrm{~nm}$ in diameter on top 


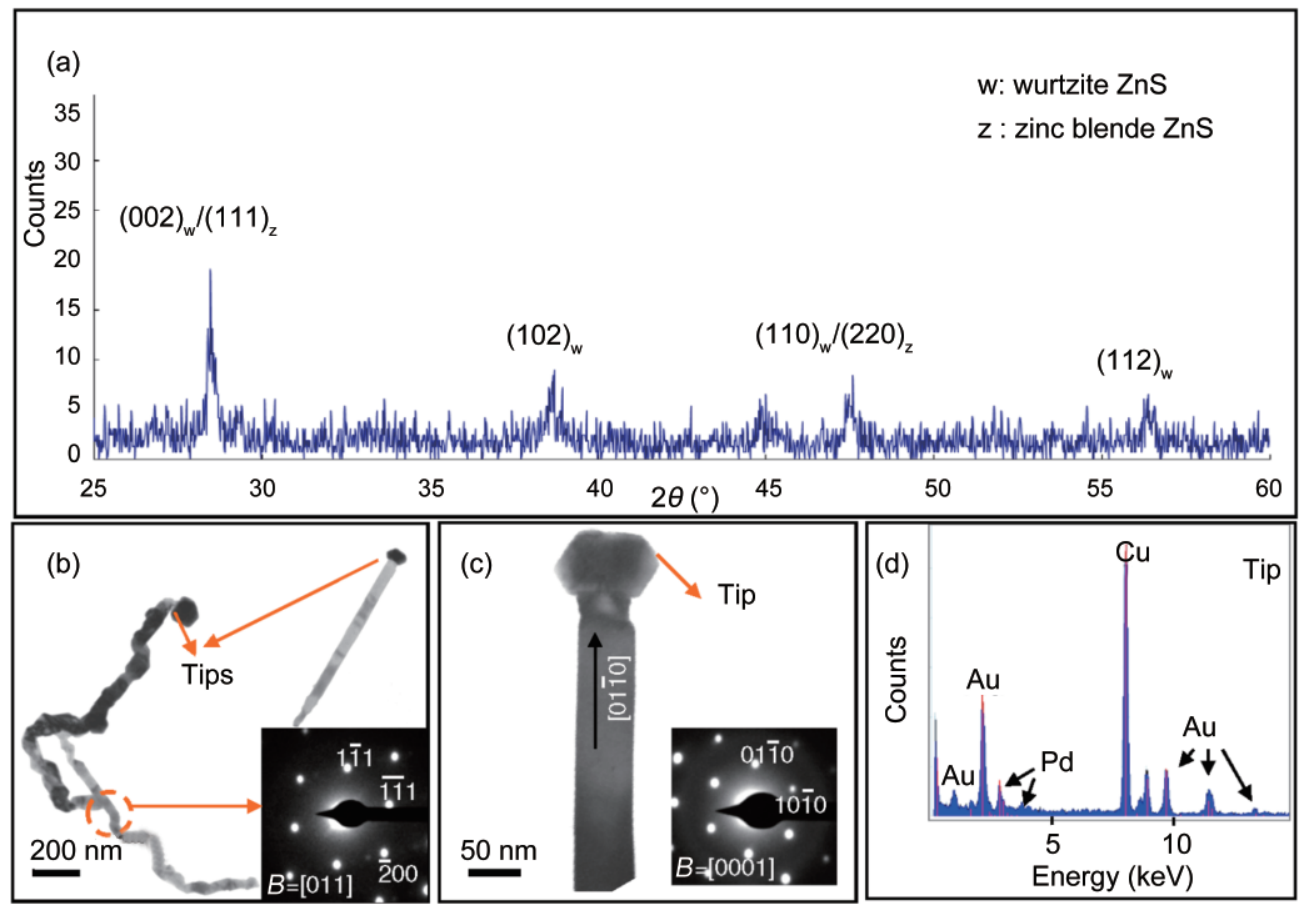

Figure 2 (a) A typical X-ray diffraction spectrum for the ZnS nanowires grown on a Si substrate. (b) A typical TEM image showing straight and curved ZnS nanowires. Inset: selected area electron diffraction pattern corresponding to a body portion of the curved ZnS nanowire shown on the left. (c) TEM image of a straight ZnS nanowire and the corresponding SAED pattern revealing its single-crystalline wurtzite structure. (d) The EDX spectrum corresponding to the tip shown in the TEM

(as indicated by the the red arrowheads). The single crystalline nature of each curved $\mathrm{ZnS}$ nanowire is identified by the inset SAED pattern in Fig. 2(b) which shows a zinc blende structure. The straight nanowire in Fig. 2(c) proved to be of wurtzite structure, as confirmed by the inset SAED diffraction pattern, with a growth direction along [0110]. The EDX spectrum shown in Fig. 2(d) further confirms the nanoparticle tips to be composed of of Au and Pd.

Under high-energy electron beam irradiation, the zinc blende-structured zigzag $\mathrm{ZnS}$ nanowires were found to be metastable in terms of morphology and structure. Figure 3(a) shows a typical TEM image of a zigzag $\mathrm{ZnS}$ nanowire with a diameter of $\sim 80 \mathrm{~nm}$, and a length of $\sim 2.5 \mu \mathrm{m}$. The SAED pattern in the inset of Fig. 3(a) corresponding to the straight portion of a zigzag $\mathrm{ZnS}$ nanowire clearly identifies its single crystal zinc blende structure and a growth direction along $[\overline{1} 1 \overline{1}]$. Figures 3(b) and 3(c) respectively show the electron irradiation-induced morphology changes of the top-left box region (the nanowiretip interface) and bottom-right box region (nanowire curved region). Pairs of TEM images compares the morphologies before (Figs. 3(b) and 3(c): top) and after (Figs. 3(b) and 3(c): bottom) irradiation under TEM (120 keV electrons, $10 \mathrm{~min}$ ). It is clearly seen that the surface of the $\mathrm{ZnS}$ nanowires became very rough compared to the originally smooth surface in both the tip-nanowire interface region (Fig. 3(b): bottom) and the nanowire body region (Fig. 3(c): bottom). The TEM EDX result corresponding to the electronirradiated nanowire-tip interface (Fig. 3(d)) clearly identifies the presence of appreciable amounts of oxygen $(\mathrm{O})$ on the $\mathrm{ZnS}$ nanowire surface in addition to the $\mathrm{Au}, \mathrm{Zn}$, and $\mathrm{S}$, which may suggest a surface oxidation-induced roughening mechanism. It is also worth noting that on the zinc blende-structured curved ZnS nanowire body, face-centered cubic (fcc)structured AuPd nanoparticles with a size of 3-5 $\mathrm{nm}$ have been observed in the high-resolution TEM image shown in Fig. 3(e), as acquired under $200 \mathrm{keV}$ TEM. The SEM image in Fig. 3(f) further confirms the presence of AuPd nanoparticles on the zigzag ZnS nanowire body, as indicated by the red arrowheads.

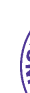




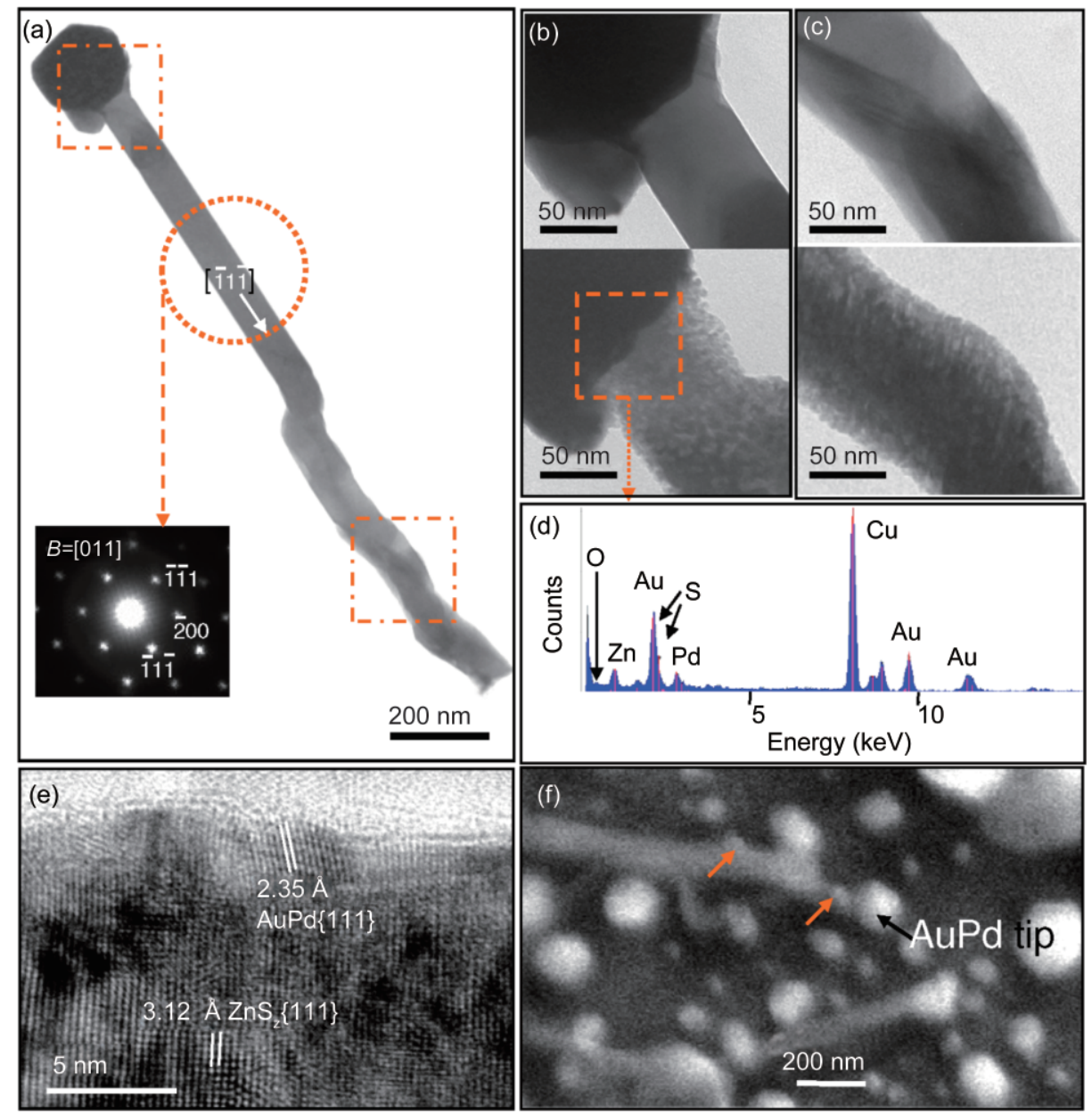

Figure 3 (a) A typical TEM image showing a zigzag ZnS nanowire $\sim 100 \mathrm{~nm}$ wide and $\sim 2.5 \mu \mathrm{m}$ long. Inset: SAED pattern corresponding to the straight portion of the nanowire. (b) and (c) TEM images corresponding to the ZnS nanowire-AuPd nanoparticle interface region (b) and curved body region (c) before (top images) and after (bottom images) significant $120 \mathrm{keV}$ electron irradiation. (d) A TEM EDX spectrum corresponding to the interface region in (b). (e) A high resolution TEM image showing FCC-structured AuPd nanoparticles on the surface of a zinc blende-structured ZnS nanowire body. (f) An SEM image showing the dispersed AuPd nanoparticles on the substrate and the nanowire body, as indicated by red arrowheads

Under higher voltage electron irradiation, the zigzag ZnS nanowire proved to be much more sensitive in terms of structure and morphology evolution. Figure 4(a) displays TEM micrographs of a single $\mathrm{ZnS}$ nanowire with different local prolonged exposures under $200 \mathrm{keV}$ electron irradiation. Regions I, II, and III on this curved ZnS nanowire were respectively exposed for long periods ( $\sim 60$ min), medium length periods ( $45 \mathrm{~min})$, and short periods ( $30 \mathrm{~min})$. To determine the structure changes during the course of the electron irradiation, SAED patterns and high resolution TEM images were recorded. A typical SAED pattern and high resolution
TEM image obtained after a short period exposure are shown in Figs. 4(b) and 4(c), respectively. An extra set of single crystalline diffraction planes was found corresponding to wurtzite-structured $\mathrm{ZnO}$, with a matched interface between $(220)_{\mathrm{ZnS}(\mathrm{z})}$ and $(10 \overline{1} 2)_{\mathrm{ZnO}(\mathrm{w})}$, with another interface between atomic planes $(1 \overline{1} 1)_{\mathrm{ZnS}(\mathrm{z})}$ and $(0002)_{\mathrm{ZnO}(\mathrm{w})}$ with a $16 \%$ lattice mismatch and a small-angle rotation. The high resolution TEM image in Fig. 4(c) clearly displays the lattices of surface oxidized $\mathrm{ZnO}$ and the zinc blende $\mathrm{ZnS}$ nanowire core. After exposure for $60 \mathrm{~min}$ to 200 $\mathrm{keV}$ electron irradiation, the solid zinc blende $\mathrm{ZnS}$ nanowire transformed into a suspended nanoparticle 
network bridging across the less-exposed remaining solid nanowire (Fig. 4(d)). The zoom-in image in Fig. 4(e) clearly reveals the network of dispersed $\sim 5-10 \mathrm{~nm}$ sized nanoparticles, with an individual nanoparticle $\sim 6 \mathrm{~nm}$ wide shown on the right. With a medium length period ( $45 \mathrm{~min}$ ) of electron exposure at $200 \mathrm{keV}$, the ZnS nanowires can be controllably transformed into $\mathrm{ZnS}-\mathrm{ZnO}$ core-shell nanocables, as illustrated in Fig. 4(f), with the formation of a $\sim 20 \mathrm{~nm}$ thick $\mathrm{ZnO}$ shell (Fig. 4(f): right) as a result of oxidation of ZnS. Exposure at $200 \mathrm{keV}$ for a short period of time ( $\sim 30 \mathrm{~min}$ ) also gave rise to appreciable surface degradation of the curved region of the $\mathrm{ZnS}$ nanowire, leading to a discontinuous $\mathrm{ZnO}$ nanofilm as illustrated in Fig. 4(g) (left). With increasing curvature in the curved region, a more discontinuous $\mathrm{ZnO}$ nanoshell was observed which could be due to local stress concentration associated with $\mathrm{ZnO} / \mathrm{ZnS}$ lattice mismatch.

As described in the above results, AuPd nanoparticle tips were found on top of each $\mathrm{ZnS}$ nanowire. Therefore, an AuPd catalyzed growth process is presumably responsible for the $\mathrm{ZnS}$ nanowire growth; this could be a vapor-liquid-solid (VLS) or a vapor-solid-solid (VSS) process. In our process, the growth temperature of $\mathrm{ZnS}$ nanowires was controlled in the range $\sim 700-900{ }^{\circ} \mathrm{C}$, whereas the melting point of $\mathrm{AuPd}(\mathrm{Au}: \mathrm{Pd}=40: 60$, weight percentage) is $\sim 1470{ }^{\circ} \mathrm{C}$ [22], much higher than $900{ }^{\circ} \mathrm{C}$. Despite the possible influence of low pressure in
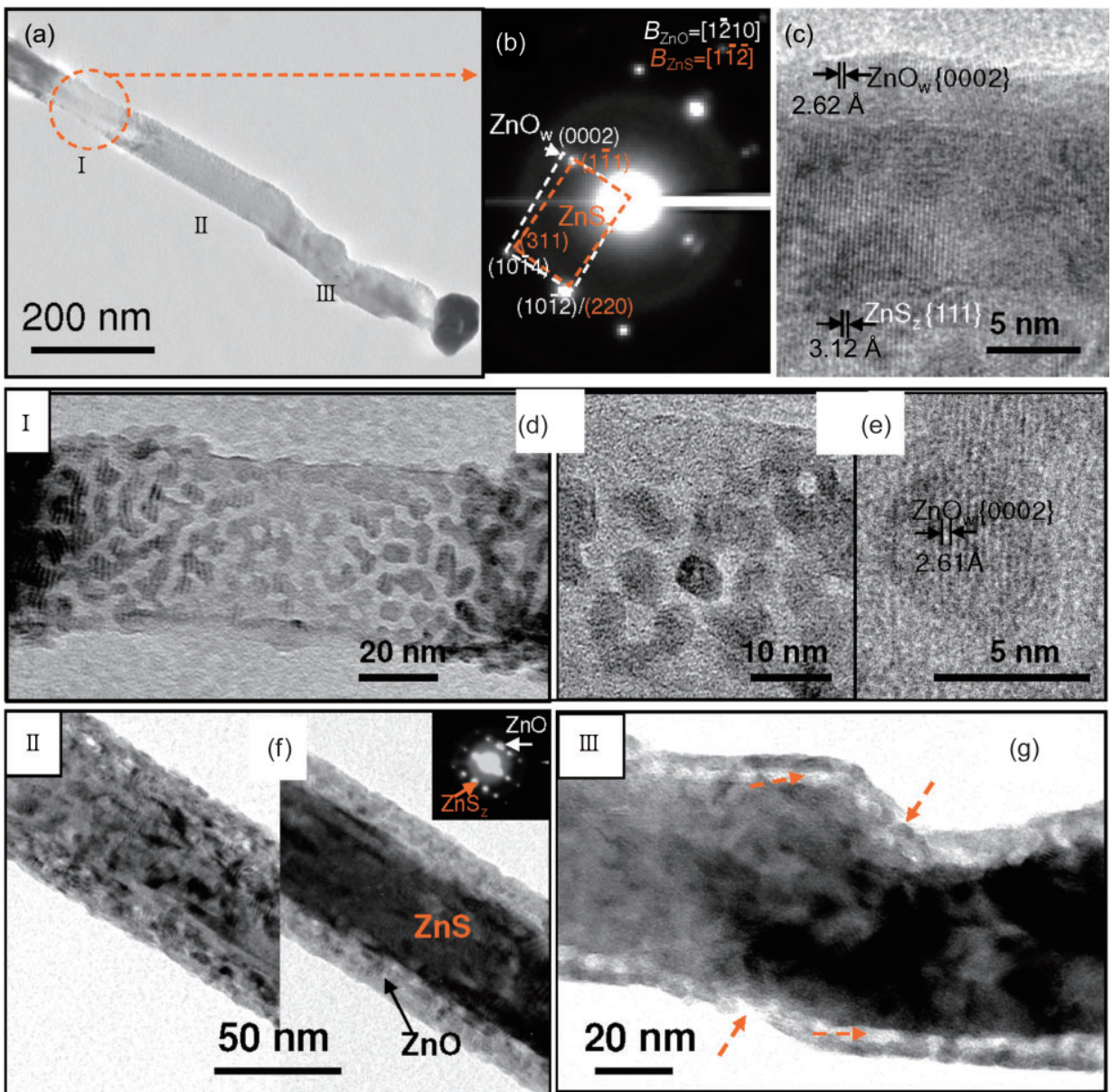

Figure 4 Structure and morphology evolution under $200 \mathrm{keV}$ electron irradiation in TEM: (a) TEM images showing a zigzag ZnS nanowire after a long period (I), a medium period (II), and a short period of exposure (III) to electron irradiation; (b) and (c) are respectively a typical SAED pattern corresponding to the zigzag ZnS nanowire body after a short period of electron irradiation and the resulting oxidized ZnS nanowire surface region; (d) and (e) are the $\mathrm{ZnO}$ nanoparticle networks formed after a long period of electron irradiation; (f) ZnS-ZnO core-shell nanocable formed after a medium period of electron irradiation; and (g) discontinuous surface oxide layer on the curved region of a ZnS nanowire after a short period of electron irradiation

\section{(1)}

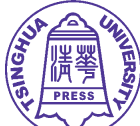


decreasing the sublimation temperature of AuPd, there is unlikely to be liquid phase AuPd present in this growth system. Furthermore, although the nanoparticle size affects the melting temperature [23], it has been shown that the melting point drastically decreases only when the particle size is smaller than $\sim 5-10 \mathrm{~nm}$, and if the particles are larger than $~ 15-$ $20 \mathrm{~nm}$, the melting point is close to that of the bulk [24]. Since the AuPd alloy nanoparticles used in this study were larger than $80 \mathrm{~nm}$ in size, the size effect is negligible. Therefore, a VSS growth process is most likely to be responsible for the growth of $\mathrm{ZnS}$ nanowires observed in this study.

In this VSS process, AuPd solid alloy nanoparticles function as nucleation sites to absorb incoming $\mathrm{ZnS}$ vapor transported by $\mathrm{N}_{2}$ gas together with oxidized carbon vapor $\left(\mathrm{CO}\right.$ or $\left.\mathrm{CO}_{2}\right)$. Due to the limited solubility of $\mathrm{ZnS}$ in solid AuPd, the precipitation of $\mathrm{ZnS}$ solute results in the formation of $\mathrm{ZnS}$ nanowires. The instant and continuous surface and bulk diffusion of ZnS solute along/across the AuPd nanoparticles ensures the growth of micronscale long nanowires, where the diameter of the nanowire is confined by the AuPd nanoparticle size and incoming flux of $\mathrm{ZnS}$ vapor. In the higher temperature region close to $900{ }^{\circ} \mathrm{C}$, larger sized AuPd nanoparticle tips were observed compared to the lower temperature region close to $700{ }^{\circ} \mathrm{C}$, as a result of easier agglomeration of AuPd through solid diffusion at higher temperature. Graphite is introduced into the growth system in order to afford a reducing atmosphere for protecting $\mathrm{ZnS}$ from oxidation. Although surface adsorbed residual oxygen has been detected in the SEM EDX results (Fig. 1(c)), TEM characterization has clearly excluded the presence of the surface oxidation product $(\mathrm{ZnO})$. The presence of AuPd nanoparticles on the $\mathrm{ZnS}$ nanowire surface might be due to the secondary evaporation and deposition of an AuPd nanoparticle film at low pressure $\left(\sim 1 \times 10^{-2} \mathrm{mbar}\right)$ and transient high temperatures $\left(\sim 700-900{ }^{\circ} \mathrm{C}\right)$ during the cooling period. On the other hand, the existence of AuPd nanoparticles on the nanowire surface confirmed by both SEM and TEM suggests a possible mechanism for the formation of the zigzag nanowires. That is to say, the "surface diffusion" of the AuPd catalyst could directly guide a kinking phenomenon similar to the surface tension effect-induced kinking in the Au-catalyzed growth of Si nanowires [25]. Multiple kinking will lead to zigzag-structured $\mathrm{ZnS}$ nanowires. The zigzag growth habit could also be facilitated by the many growth direction switches amongst the multiple equivalent $\langle 111\rangle$ directions. In contrast, in the case of wurtzite-structured nanowires, the lack of equivalent directions for switching growth is a possible reason why the wurtzite $\mathrm{ZnS}$ nanowires are straight.

The results show that the zinc blende zigzag nanowires are metastable. Density functional theory (DFT) calculations within the generalized gradient approximation (GGA) revealed that the total energy difference between the wurtzite and zinc blende bulk structures is $5.6 \mathrm{meV} /$ atom, with zinc blende $\mathrm{ZnS}$ being the more stable. However, for nanostructures with small diameters, i.e., ZnS nanowires, the wurtzite structure is more stable than the zinc blende structures [26].

The degradation of $\mathrm{ZnS}$ nanowires under electron irradiation can be attributed to three factors. First, the heating effect due to high energy electron irradiation promotes the reaction of surface $\mathrm{ZnS}$ atoms with residual oxygen present in the TEM column despite the high vacuum conditions. Under higher voltage TEM operation, higher energy densities lead to greater heating effects resulting in higher instantaneous temperatures, and this leads to faster degradation and structure/morphology transformation of $\mathrm{ZnS}$ nanowires into $\mathrm{ZnS}-\mathrm{ZnO}$ coreshell nanocables [15], and $\mathrm{ZnS}-\mathrm{ZnO}$ nanoparticle networks. Secondly, the SEM characterization suggests the possible facile adsorption of oxygen on the $\mathrm{ZnS}$ surface, leading to easy onset of oxygen substitution into the $\mathrm{ZnS}$ surface lattice upon heat, i.e., nanoscale surface oxidation. Thirdly, as described in Figs. 3(e) and 3(f), fine AuPd nanoparticles ( 5 nm) are dispersed on the surface of the zinc blende $\mathrm{ZnS}$ nanowire body. These AuPd nanoparticles are good oxidation catalysts, and therefore could function as strong oxygen adsorption sites and promote oxidation of the ZnS nanowire surface, and therefore induce a fast and sensitive structure evolution under electron irradiation. 


\section{Conclusions}

Large scale single crystal $\mathrm{ZnS}$ nanowires have been successfully fabricated during an AuPd-catalyzed vapor-solid-solid process. Two types of single crystal ZnS nanowire are formed: a large fraction of zigzag zinc blende nanowires and a small fraction of straight wurtzite nanowires. The zigzag zinc blende single crystal $\mathrm{ZnS}$ nanowires are metastable as shown by high energy electron beam irradiation under a transmission electron microscope. With relatively low voltage electron irradiation, surface oxidation occurs, leading to a wurtzite-structured $\mathrm{ZnO}$ nanoparticle layer which roughens the surface of the zigzag zinc blende ZnS nanowire. When higher voltage electron irradiation was applied, local regions of $\mathrm{ZnS}$ nanowires were transformed into $\mathrm{ZnO}-\mathrm{ZnS}$ nanocables or dispersed $\mathrm{ZnO}-\mathrm{ZnS}$ nanoparticles, depending on the irradiation time. AuPd nanoparticles also appeared on the surface of the zigzag ZnS nanowire body, which might have a catalytic effect and speed up the oxidative structure evolution process of $\mathrm{ZnS}$ nanowires under electron irradiation. The possibility of deliberate modification of their structure under electron irradiation means that the nanostructures reported here are likely to be useful for fabricating optoelectronic devices with tunable functions.

\section{Acknowledgements}

The authors are grateful for the financial support from the University of Connecticut New Faculty start-up funds, and the University of Connecticut Large Faculty Research Grant. Acknowledgement is also made to the Donors of the American Chemical Society Petroleum Research Fund for partial support of this research.

\section{References}

[1] Ahn, H. S.; Lee, K. R.; Kim, D. Y.; Han, S. W. Field emission of doped carbon nanotubes. Appl. Phys. Lett. 2006, 88, 93122.

[2] Jo, S. H.; Lao, J. Y.; Ren, Z. F.; Farrer, R. A.; Baldacchini, T.; Fourkas, J. T. Field-emission studies on thin films of zinc oxide nanowires. Appl. Phys. Lett. 2003, 83, 4821.

[3] Ha, B.; Seo, S. H.; Cho, J. H.; Yoon, C. S.; Yoo, J.; Yi, G. C.; Park, C. Y.; Lee, C. J. Optical and field emission properties of thin single-crystalline GaN nanowires. J. Phys. Chem. B. 2005, 109, 11095-11099.

[4] He, J. H.; Yang, R. S.; Chueh, Y. L.; Chou, L. J.; Chen, L. J.; Wang, Z. L. Aligned AIN nanorods with multi-tipped surfaces-growth, field-emission, and cathodoluminescence properties. Adv. Mater. 2006, 18, 650-654.

[5] Bredol, M.; Merikhi, J. Structure- and size-controlled ultrafine ZnS nanowires. J. Mater. Sci. 1998, 33, 471476.

[6] Kar, S.; Biswas, S.; Chaudhuri, S. Catalytic growth and photoluminescence properties of $\mathrm{ZnS}$ nanowires. Nanotechnology 2005, 16, 737-740.

[7] Fang, X.; Ye, C.; Zhang, L.; Wang, Y.; Wu, Y. Temperature-controlled catalytic growth of ZnS nanostructures by the evaporation of ZnS nanopowders. Adv. Funct. Mater. 2005, 15, 63-68.

[8] Moon, H.; Nam, C.; Kim, C.; Kim, B. Synthesis and photoluminescence of zinc sulfide nanowires by simple thermal chemical vapor deposition. Mater. Res. Bull. 2006, 41, 2013-2017.

[9] Moore, D.; Wang, Z. L. Growth of anisotropic onedimensional ZnS nanostructures. J. Mater. Chem. 2006, 16, 3898-3905.

[10] Chang, Y.; Wang, M.; Chen, X.; Ni, S.; Qiang, W. Field emission and photoluminescence characteristics of ZnS nanowires via vapor phase growth. Solid State Comm. 2007, 142, 295-298.

[11] Fan, X.; Meng, X.; Zhang, X.; Wu, S.; Lee, S. Formation of $\mathrm{ZnS} / \mathrm{SiO}_{2}$ nanocables. Appl. Phys. Lett. 2005, 86, 173111.

[12] Jiang, Y.; Zhang, W. J.; Jie, J. S.; Meng, X. M.; Zapien, J. A.; Lee, S. Homoepitaxial growth and lasing properties of ZnS nanowire and nanoribbon arrays. Adv. Mater. 2006, 18, 1527-1532.

[13] Zhang, X.; Zhang, Y.; Song, Y.; Wang, Z.; Yu, D. Optical properties of ZnS nanowires synthesized via simple physical evaporation. Physica E 2005, 28, 1-6.

[14] Geng, B. Y.; Liu, X. W.; Du, Q. B.; Wei, X. W.; Zhang, L. D. Structure and optical properties of periodically twinned ZnS nanowires. Appl. Phys. Lett. 2006, 88, 163104.

[15] Yan, J.; Fang X. S.; Zhang, L.; Bando, Y.; Dierre, B.; Sekiguchi, T.; Gautam, U. K.; Golberg, D. Structure 
and cathodoluminescence of individual $\mathrm{ZnS} / \mathrm{ZnO}$ biaxial nanobelt heterostructures. Nano Lett. 2008, 8, 27942799.

[16] Fang, X. S.; Bando, Y.; Liao, M. Y.; Gautam, U. K.; Zhi, C. Y.; Dierre, B.; Liu, B. D.; Zhai, T. Y.; Sekiguchi, T.; Koide, Y.; Golberg, D. Single-crystalline ZnS nanobelts as ultraviolet-light sensors. Adv. Mater. 2009, 21, 20342039.

[17] Ding, Y.; Wang, X. D.; Wang, Z. L. Phase controlled synthesis of ZnS nanobelts: Zinc blende vs. wurtzite. Chem. Phys. Lett. 2004, 398, 32-36.

[18] Pan, Y. W.; Yu, J.; Hu, Z.; Li, H. D.; Cui, Q. L.; Zou, G. T. Pressure-induced structural transitions of the zinc sulfide nano-particles with different sizes. J. Mater. Sci. Technol. 2007, 23, 193-195.

[19] Meng, X. M.; Liu, J.; Jiang, Y.; Chen, W. W.; Lee, C. S.; Bello, I.; Lee, S. T. Structure- and size-controlled ultrafine ZnS nanowires. Chem. Phys. Lett. 2003, 382, 434-438.

[20] Li, Q.; Wang, C. Fabrication of Zn/ZnS nanocable heterostructures by thermal reduction/sulfidation. Appl. Phys. Lett. 2003, 82, 1398.
[21] Shi, L.; Xu, Y. M.; Li, Q.; Wu, Z. Y.; Chen, F. R.; Kai, J. J. Single crystalline $\mathrm{ZnS}$ nanotubes and their structural degradation under electron beam irradiation. Appl. Phys. Lett. 2007, 90, 211910.

[22] Massalski, T. B. Binary Alloy Phase Diagrams, 2nd Ed.; ASM International: Materials Park, OH, 1990; Vol. 1.

[23] Buffat, P.; Borel, J. P. Size effect on the melting temperature of gold particles. Phys. Rev. A 1976, 13, 2287-2298.

[24] Campos, L. C.; Tonezzer, M.; Ferlauto, A. S.; Grillo, V.; Magalhães-Paniago, R.; Oliveira, S.; Ladeira, L. O.; Lacerda. R. G. Vapor-solid-solid growth mechanism driven by epitaxial match between solid AuZn alloy catalyst particles and $\mathrm{ZnO}$ nanowires at low temperatures. Adv. Mater. 2008, 20, 1499-1504.

[25] Wagner, R. S.; Doherty, C. J. Mechanism of branching and kinking during VLS crystal growth. J. Electrochem. Soc. 1968, 115, 93-99.

[26] Chen, H.; Shi, D.; Qi, J.; Jia, J.; Wang, B. The stability and electronic properties of wurtzite and zinc-blende ZnS nanowires. Phys. Lett. A 2009, 373, 371-375. 